\title{
Partial breast radiotherapy with simple teletherapy techniques
}

\author{
Gábor Fekete, Dóra Újhidy, Zsófia Együd, Laura Kiscsatári, Gusztáv Marosi, \\ Zsuzsanna Kahán, and Zoltán Varga
}

Department of Oncotherapy, University of Szeged, Szeged, Hungary

\section{A R T I C L E I N F O}

\section{Article history:}

Received 17 June 2014

Received in revised form

16 February 2015

Accepted 19 March 2015

\section{Keywords:}

Electron beam

Fractionation

Partial breast radiotherapy

Photon beam

Acute skin toxicity

Late skin toxicity

\begin{abstract}
A B S T R A C T
A prospective pilot study of partial breast irradiation (PBI) with conventional vs hypofractionated schedules was set out. The study aimed to determine efficacy, acute and late side effects, and the preference of photon vs electron irradiation based on individual features. Patients were enrolled according to internationally accepted guidelines on PBI. Conformal radiotherapy plans were generated with both photon and electron beams, and the preferred technique based on dose homogeneity and the radiation exposure of healthy tissues was applied. For electron dose verification, a special phantom was constructed. Patients were randomized for fractionation schedules of $25 \times 2$ vs $13 \times 3 \mathrm{~Gy}$. Skin and breast changes were registered at the time of and $\geq 1$ year after the completion of radiotherapy. Dose homogeneity was better with photons. If the tumor bed was located in the inner quadrants, electron beam gave superior results regarding conformity and sparing of organ at risk (OAR). If the tumor was situated in the lateral quadrants, conformity was better with photons. A depth of the tumor bed $\geq 3.0 \mathrm{~cm}$ predicted the superiority of photon irradiation (odds ratio $[\mathrm{OR}]=23.6,95 \% \mathrm{CI}: 5.2$ to 107.5 , $p<0.001$ ) with $>90 \%$ sensitivity and specificity. After a median follow-up of 39 months, among 72 irradiated cases, 1 local relapse out of the tumor bed was detected. Acute radiodermatitis of grade I to II, hyperpigmentation, and telangiectasia developed $\geq 1$ year after radiotherapy, exclusively after electron beam radiotherapy. The choice of electrons or photons for PBI should be based on tumor bed location; the used methods are efficient and feasible.
\end{abstract}

(c) 2015 American Association of Medical Dosimetrists.

\section{Introduction}

Radiotherapy is an integral part of breast-conserving surgery, which lowers the risk of both local recurrence and breast cancerrelated death. ${ }^{1}$ In low-risk breast cancer, accelerated partial breast radiotherapy (APBI) is widely practiced and is an equally efficient alternative to whole-breast radiotherapy (WBI). ${ }^{2-4}$ Guidelines for patient selection have been reported by the American Society for Radiation Oncology and the Groupe Européen de CuriethérapieEuropean Society for Therapeutic Radiology and Oncology, 5,6 although a trend toward widening eligibility criteria is seen., ${ }^{2,3,7}$

There are multiple delivery methods for APBI, such as intraoperative radiotherapy, intracavitary/interstitial brachytherapy, and external beam radiotherapy. ${ }^{3}$ Regarding effectiveness, similarly low ipsilateral recurrence rates are reported with the 3 methods. However, there is controversy regarding cosmetic outcome after teletherapy, which is probably related to technical details. ${ }^{8}$

Reprint requests to: Zoltán Varga, Korányi fasor 12, H-6720 Szeged, Hungary.

E-mail: varga.zoltan@med.u-szeged.hu
Although robust and long-term experiences were collected with brachytherapy and intraoperative radiotherapy, as the access to these methods is limited, the use of external beam radiotherapy is increasing. For teletherapy, both conformal radiotherapy (CRT) and intensity-modulated radiotherapy (IMRT) have been used ${ }^{2,3,6,8,9}$; the advantages, as compared with brachytherapy, are noninvasivity, wide availability, and cost efficiency. A potential advantage of CRT in terms of cosmetic outcome (clearly adversely affected by skin or subcutaneus fibrosis, skin atrophy, discoloration, and telangiectasia) could be the more homogeneous dose distribution as compared with that with brachytherapy. ${ }^{10}$ Besides having less and shorter experience, additional disadvantages of CRT are the lack of optimal fractionation schedule and the greater dose to the organs at risk (OARs) including uninvolved tissue of the target breast. ${ }^{3}$ Dose homogeneity is even better with IMRT than with CRT. ${ }^{11}$ IMRT was expected to improve cosmetic outcome and to further decrease OAR exposure. Nevertheless, some trials gave negative results. ${ }^{12-15}$ The study by Jagsi et al. ${ }^{12}$ was prematurely terminated because 7 of 32 patients showed unacceptable cosmetic results 2.5 years after deep inspiration breath-hold IMRT, 
which increased to $26.7 \%$ of the cases after 5 years. ${ }^{13}$ Hepel et $a l^{14}$ reported that, at a median follow-up of 15 months following CRT, 25\% of the patients showed moderate to severe and $8.3 \%$ showed severe subcutaneous fibrosis. In the large Randomized Trial of Accelerated Partial Breast Irradiation (RAPID) clinical trial, after CRT APBI, significantly poorer cosmetic outcome was reported owing to late radiation toxicity, than in the WBI arm. ${ }^{15}$ In contrast, based on the interim results of another similar large prospective randomized trial, the NSABP B39/RTOG 0413 study, no safety issue emerged. ${ }^{16}$ Then, 2 prospective ${ }^{8,9}$ and 1 retrospective ${ }^{17}$ IMRT studies indicated excellent efficacy and cosmetic outcome. The head-to-head comparison of CRT (poorer dose homogeneity but less irradiated healthy tissue overall) and IMRT (superior dose homogeneity but more healthy tissue irradiated with low doses), with special regard to skin changes and breast fibrosis determining cosmetic results, is still awaited.

There is a need to provide partial breast irradiation (PBI) on a routine basis to an increasing number of patients with low-risk breast cancer, even in places with limited technical background. In this pilot study, we aimed at prospectively investigating the feasibility of PBI using photon vs electron irradiation with conventional vs accelerated schedules. As previously no experience had been gained with hypofractionated breast radiotherapy, we tested the feasibility of 3-Gy fraction dose irradiation (either with photon or electron beams) to the relatively small PBI breast volumes, in comparison with the conventional fractionation schedule. Besides the analysis of efficacy and acute and late side effects, we intended to find out which parameters could predict the preference of electron over photon irradiation in everyday practice.

\section{Methods and Materials}

The study was approved by the Institutional Review Board of the University of Szeged. All participants gave their written informed consent. Patients after breastconserving surgery were eligible if they were $>50$ years of age, had unifocal carcinoma not larger than $30 \mathrm{~mm}$ of nonlobular histologic type, had node-negative disease, had absence of extensive intraductal component (EIC) or in situ ductal cancer (DCIS), had lymphovascular invasion, had surgical margin $\geq 2 \mathrm{~mm}$, or had surgical clips present for the identification of the tumor bed. In addition, the situation of the tumor bed within the breast and the adjuvant systemic therapy given according to the institutional guidelines were registered. Patients were checked at the time of the radiotherapy, 3 months after completion, and every 6 months thereafter. Skin or breast changes were evaluated on the first and the last day of the radiotherapy and $\geq 1$ year thereafter according to the Common Terminology Criteria for Adverse Events (CTC AE) vs 3.0 system.

\section{Radiotherapy techniques}

Computed tomography-based 3-dimensional treatment planning (Oncentra Masterplan ( $\mathrm{V} 4.3$, Nucletron) and CRT were performed in all cases with the patient in the supine position. All relevant technical details about patient positioning, immobilization, and imaging have been published previously. ${ }^{18}$ The target volumes were contoured on the computed tomography slices in the radiotherapy planning system by placing a $1-\mathrm{cm}$ margin around the surgical cavity as defined by the surgical clips (clinical target volume), and using a $0.5-\mathrm{cm}$ planning target volume (PTV) margin, but excluding the chest wall and the tissue $4 \mathrm{~mm}$ under the skin. ${ }^{19}$ The OARs were contoured as previously described ${ }^{20}$; the part of the operated breast excluding the PTV was indicated as "ipsilateral breast." The size of the PTV and the distance of its geometric center beneath the skin (D) were recorded. The D was measured in the radiotherapy planning system by defining a simple isocentric photon beam to the center of the PTV, and setting up the gantry angle perpendicular to the surface. The D was $100 \mathrm{~cm}$ minus the focus-skin distance. The patients were randomized to receive a mean dose to the PTV of either $25 \times 2$ or $13 \times 3 \mathrm{~Gy}$. Both electron and photon plans were generated, and the choice of radiotherapy technique was based on dose distribution in the PTV and OAR exposure.

Photon beams: In total, two 6- or 15-MV photon fields were used with a physical wedge of $30^{\circ}$ conformed to the PTV with a 58-leaf multileaf collimator Occasionally, 1 additional segment was used for better dose homogeneity. The dose calculation was performed by the collapsed cone algorithm.

Electron beams: Overall, 6-, 9-, 12-, or 15-MeV (occasionally mixed) electron beams using an applicator of $10 \times 10 \mathrm{~cm}$ and rectangular inserts $(6 \times 6 \mathrm{~cm}, 6 \times$ $8 \mathrm{~cm}$, etc.) were applied. The dose calculation was performed by the Voxel Monte Carlo algorithm.

For the analysis of 3-dimensional dose distribution, the volume of the PTV receiving $95 \%$ to $107 \%$ of the prescibed dose $\left(V_{95 \%}\right.$ to $\left.V_{107 \%}\right)$, and the doses received by $5 \%$ and $95 \%$ of the PTV ( $D_{5 \%} / D_{95 \%}$ ) were registered; the healthy tissue conformity index $(\mathrm{HTCl})$ and the conformation number $(\mathrm{CN})$ were calculated using the following equations: $\mathrm{HTCI}=\frac{\mathrm{TV}_{\mathrm{RI}}}{\mathrm{V}_{\mathrm{RI}}}$ and $\mathrm{CN}=\frac{\mathrm{TV}_{\mathrm{RI}}}{\mathrm{TV}} \times \frac{\mathrm{TV}_{\mathrm{RI}}}{\mathrm{V}_{\mathrm{VI}}}$ (TV is target volume, i.e., PTV; $\mathrm{TV}_{\mathrm{PD}}$ is target volume covered by the prescribed dose; and $\mathrm{V}_{\mathrm{PD}}$ is volume of the prescribed dose). ${ }^{20,21}$ For comparison, the relative doses to the OARs were recorded as follows: the volumes of the ipsilateral breast receiving $>100 \%, 50 \%$,

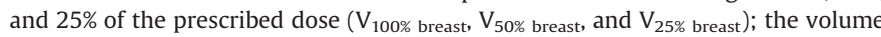
of the ipsilateral lung receiving $>40 \%$ of the prescribed dose $\left(\mathrm{V}_{40 \%}\right.$ lung $)$; the volume of the heart receiving $>50 \%$ of the prescribed dose ( $V_{50 \%}$ heart, left-sided cases); the volume of the left anterior descendent (LAD) receiving $>20 \%$ of the prescribed dose ( $\mathrm{V}_{20 \%}$ LAD, left-sided cases); and the volume of the contralateral breast receiving $>10 \%$ of the prescribed dose $\left(\mathrm{V}_{10 \%}\right.$ opp breast $)$.

The radiotherapy was delivered with a Siemens Primus linear accelerator in 5 fractions per week.

\section{Verification of the dose calculation for electron beams}

Besides routine physical quality-control measurements, for electron dose verification, a special phantom was constructed, which simulated the anatomic situation with respect to the curved surface and the structures with significantly different densities near the target volume. ${ }^{22}$ Disk-shaped aluminum and cork pieces of $5 \times 1 \mathrm{~cm}$ have been inserted into a poly(methyl methacrylate) slab

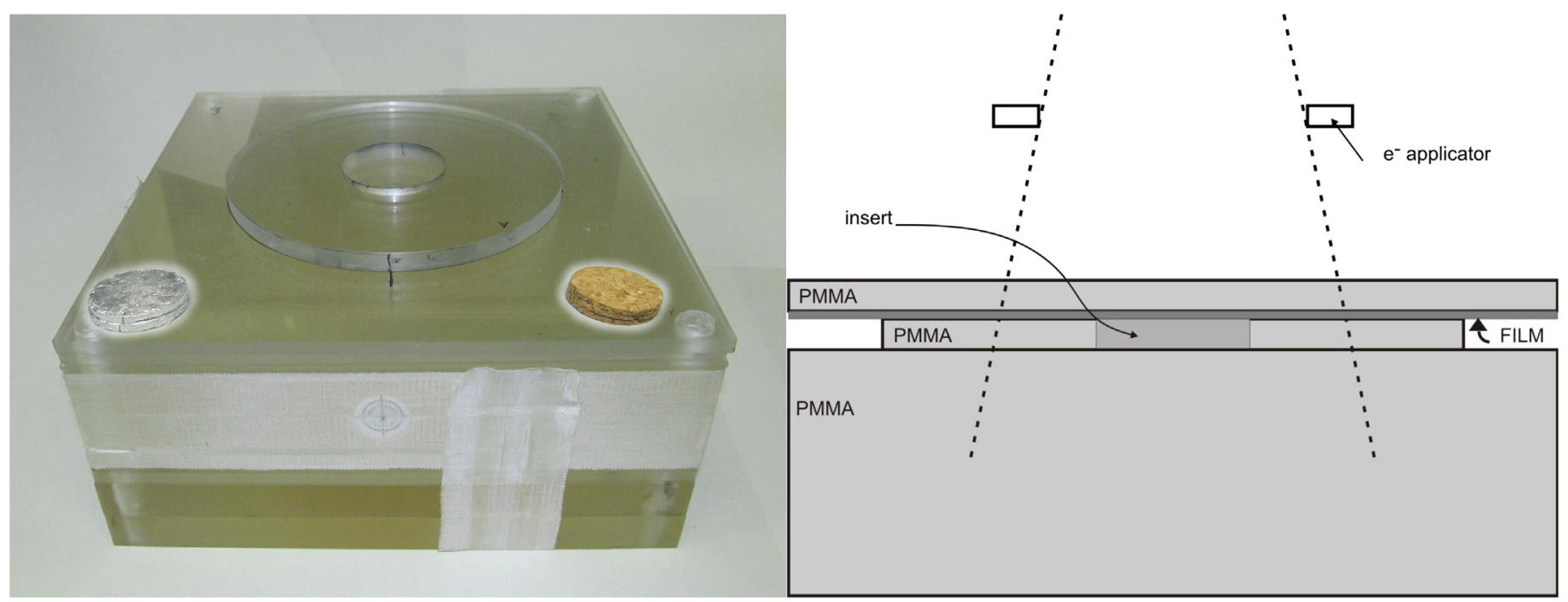

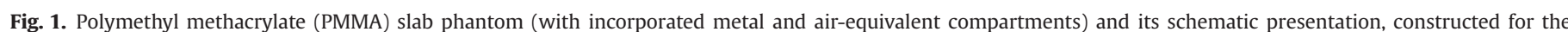
verification of electron dose calculations. (Color version of figure is available online.) 
Table 1

Patient characteristics $(n=72)$

\begin{tabular}{ll}
\hline Age (mean \pm SE, y) & $61.3 \pm 0.9$ \\
Premenopausal & $8(11.1 \%)$ \\
Menopausal & $64(88.9 \%)$ \\
Pathologic tumor size (pT) (mean \pm SE, mm) & $11.5 \pm 0.5$ \\
Invasive ductal carcinoma not special type & $63(87.5 \%)$ \\
Tubular carcinoma & $3(4.2 \%)$ \\
Mucinous carcinoma & $2(2.7 \%)$ \\
Medullary carcinoma & $1(1.4 \%)$ \\
Papillary carcinoma & $1(1.4 \%)$ \\
Mixed carcinoma & $1(1.4 \%)$ \\
Cribriform carcinoma & $1(1.4 \%)$ \\
Grade I & $27(37.5 \%)$ \\
Grade II & $34(47.2 \%)$ \\
Grade III & $11(15.3 \%)$ \\
Hormone receptor positive & $62(86.1 \%)$ \\
Hormone receptor negative & $10(13.9 \%)$ \\
HER2 negative & $70(97.2 \%)$ \\
HER2 positive & $2(2.8 \%)$ \\
Surgical margin (mean \pm SE, mm) & $7.3 \pm 0.5$ \\
Tumor location: inner quadrants & $33(45.8 \%)$ \\
Tumor location: outer quadrants & $39(54.2 \%)$ \\
Adjuvant hormone therapy & \\
$\quad$ Tromatase inhibitor & $9(12.5 \%)$ \\
Adjumoxifen & $7(9.7 \%)$ \\
\hline & $3(4.1 \%)$ \\
\hline
\end{tabular}

$\mathrm{SE}=$ standard error.

phantom with the proximal interface positioned at the depth of the maximum dose (1.2 $\mathrm{cm}$ for the used 6-MeV electron beam) (Fig. 1). Computed tomography scans of this phantom were acquired with a slice thickness of $1 \mathrm{~mm}$. Dose distribution was measured with Kodak EDR2 film in both the homogeneous and inhomogeneous cases, for normal and oblique beam incidence. Measured and calculated relative dose distributions agreed $<1 \%$ in all cases. The agreement between the calculated and measured absorbed doses in the proximal interface was within $2.5 \%$ for both aluminum and cork, with values decreasing within the accuracy threshold of film dosimetry method if applied for absolute dose estimation. Monitor unit calculations were in $<2 \%$ difference range. Thus, the Voxel Monte Carlo algorithm was found highly accurate in such a complex anatomic situation as breast radiotherapy.

\section{Statistical methods}

The differences of dose homogeneity, conformity index, and the dose-volume parameters of the OARs between the 2 irradiation techniques were examined by paired sample t-test. The associations between the various dosimetric data and the geography and the depth of the tumor bed or the irradiation technique were examined with paired sample t-tests. The incidence of acute and late toxicity was analyzed by $\chi^{2}$ test. For testing the association between the depth of the tumor bed and dose homogeneity index, correlation-regression analysis was done. To define the cutoff point for $\mathrm{D}$ and to choose the electron irradiation technique, receiver operating characteristics analysis was applied. Logistic regression models were applied to examine the potential predictors for beam selection. First, binary univariate logistic regression models were used separately, followed by the multivariate logistic regression model to examine the joint effects and interactions. A stepwise procedure was employed with a likelihood ratio test. SPSS version 20.0 for Windows (SPSS Inc., Chicago, IL) was applied for statistical analysis.

\section{Results and Discussion}

\section{Radiotherapy data}

Between November 2009 and December 2012, 72 patients were enrolled, all qualified for the inclusion criteria (Table 1). Most of the cases (79.2\%) had sentinel lymph node biopsy.

The number of the fractionation schedules and chosen radiotherapy techniques are shown in Fig. 2. Electron beams were applied in more than half of the cases. The mean \pm standard error of the PTV volume was $87.4 \pm 5.8 \mathrm{~cm}^{3}$ (36 to $308 \mathrm{~cm}^{3}$ ) (inner $v s$

\section{$\mathrm{n}=72$}

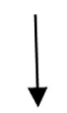

Randomization

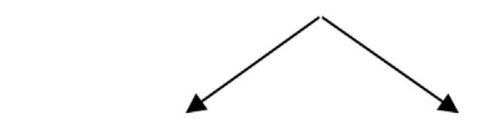

$13 x 3$ Gy

$25 \times 2$ Gy

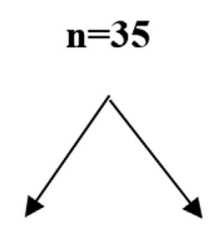

electron

$\mathbf{n}=\mathbf{2 0}$

\section{photon$$
\mathbf{n}=\mathbf{1 5}
$$

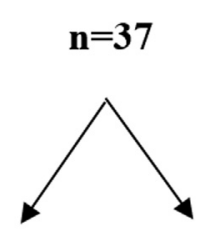

electron

$n=19$ photon $\mathrm{n}=18$
Fig. 2. Consort diagram of patients randomized to different treatment arms.

outer quadrants: 74.9 vs $98.1 \mathrm{~cm}^{3}$, respectively, $\left.p=0.043\right)$. The mean \pm standard error of D was $3.1 \pm 0.2 \mathrm{~cm}(1.0$ to $7.0 \mathrm{~cm})$ (inner quadrants: $2.1 \pm 0.1 \mathrm{~cm}$, outer quadrants: $4.0 \pm 0.2 \mathrm{~cm}, p<0.001)$. The used radiotherapy technique was selected primarily by dose distribution, and the dose to the OARs was checked thereafter. Typical field arrangements and dose distributions are shown in Fig. 3.

Dose distribution and OAR exposure were primarily determined by the location of the tumor bed (Tables 2 and 3). If the tumor was located in the inner quadrants, although the dose homogeneity was somewhat better with photon beams, the degree of conformity appeared similar regardless of beam quality (electron $v s$ photon). In contrast, if the tumor was excised from the outer quadrants, the photon plans were superior in terms of both dose homogeneity and conformity indices. In the inner-quadrant cases, the radiation exposure of the ipsilateral and the contralateral breast was more favorable with electron beams; however, no such differences existed for the lung and the LAD (Table 3). In the outer-quadrant cases, although the dose to the heart and the LAD was similar, the $\mathrm{V}_{100 \%}$ breast was significantly higher if electron

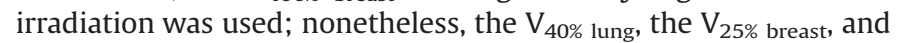
$\mathrm{V}_{50 \%}$ breast were superior with electrons (Table 3 ). Regarding the association between dose homogeneity $\left(V_{95 \%}\right.$ to $\left.V_{107 \%}\right)$ and the $D$, a strong positive correlation existed if photon beams were used $(R=$ $0.622, p<0.001$ ), and a strong negative correlation existed if electron ( $R=-0.875, p<0.001$ ) beams were used. Notably, using electrons, the $V_{95 \%}$ to $V_{107 \%}$ was unacceptably low if $D$ was $\geq 3.0 \mathrm{~cm}$ (as provided by receiver operating characteristic curve analysis); using this cutoff value, the sensitivity and specificity of the prediction were $92.3 \%$ and $90.9 \%$, respectively.

Consequently, the tumor location, the size of the PTV, and the D were first studied in binary univariate logistic regression models. Significant positive associations were found between the superiority of electron over photon irradiation and the situation of the tumor bed to the inner quadrants (odds ratio $[\mathrm{OR}]=33.3,95 \% \mathrm{CI}$ : 8.2 to $135.3, p<0.001)$, a smaller $\mathrm{D}(\mathrm{OR}=23.6,95 \% \mathrm{CI}: 5.2$ to 107.5, $p<0.001$ ), and a smaller size of the PTV (OR $=1.02,95 \% \mathrm{CI}$ : 1.0 to $1.03, p=0.01$ ). The joint effects of the quadrant, the PTV, and the $\mathrm{D}$ were examined in a multiple logistic regression model using a stepwise algorithm. Only the D remained significant in the model. 

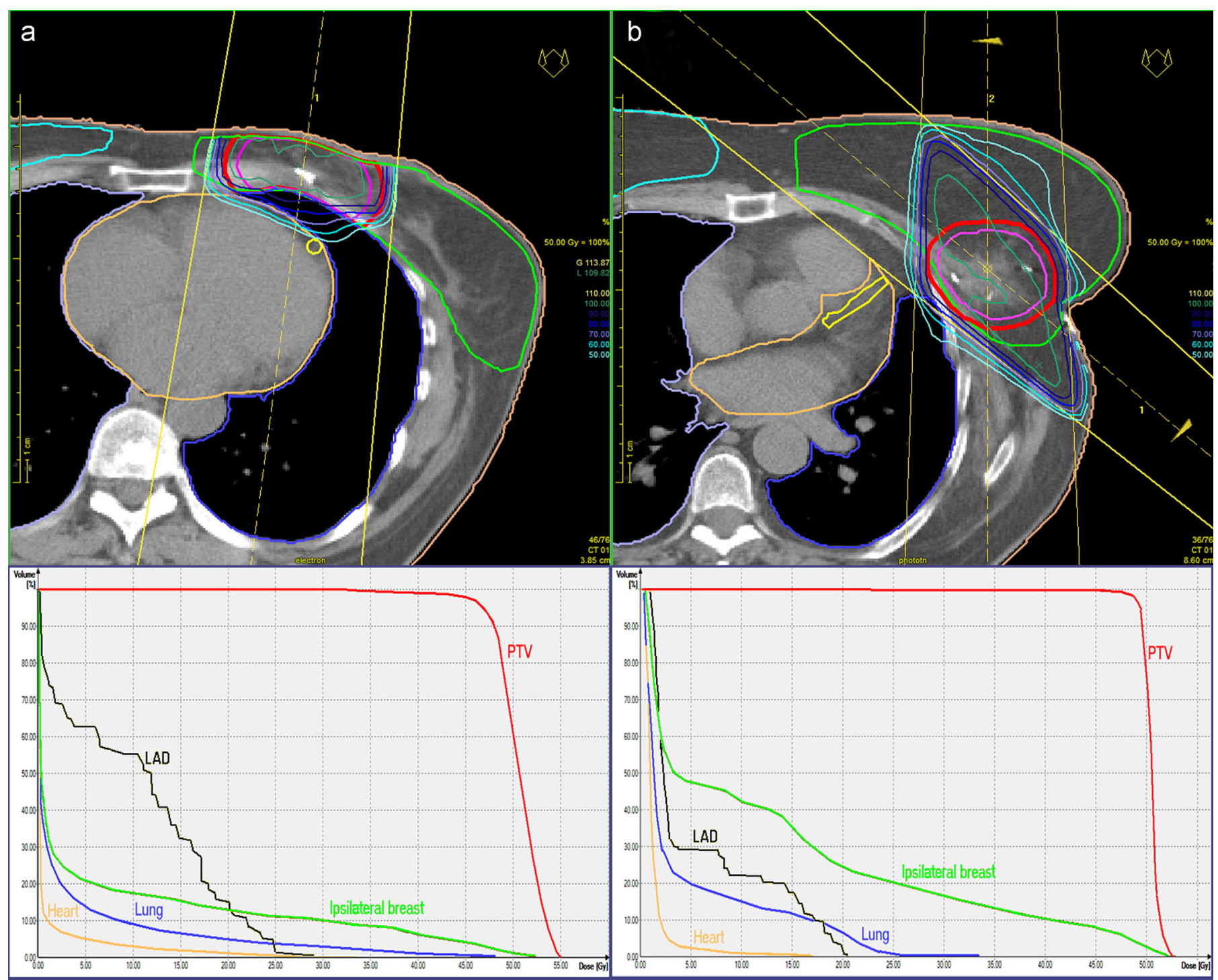

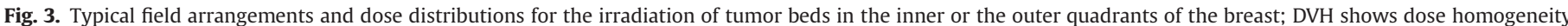

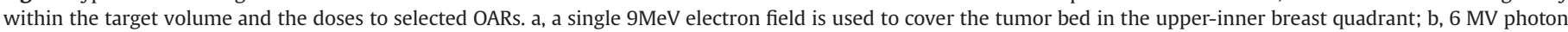
fields are applied to irradiate the tumor bed in the outer-upper quadrant of the left breast. (Color version of figure is available online.)

\section{Clinical outcome}

After a median follow-up time of 38.8 (5.6 to 45.3) months, 1 patient in the $25 \times 2$ Gy photon arm developed an in-breast relapse outside of the tumor bed, and 1 sentinel node-negative patient was diagnosed with axillary node metastasis. A patient developed and died because of distant metastases. In addition, 2 other patients died owing to other causes than breast cancer.
Skin and breast changes according to the type of radiotherapy and fractionation schedule are shown in Table 4. Acute radiodermatitis was more frequent in patients in the electron arms; hyperpigmentation and teleangiectasia developed exclusively in the electron-irradiated patients $>1$ year after the radiotherapy, in $10 \%$ and $17 \%$ of the cases, in the $25 \times 2 \mathrm{~Gy}$ and $13 \times 3 \mathrm{~Gy}$ arms, respectively. A single case of subcutaneous/breast fibrosis was detected in the $13 \times 3$ Gy photon radiotherapy arm. Breast pain

Table 2

Dose homogeneity and conformity indices according to tumor bed localization (inner vs outer quadrant)

\begin{tabular}{|c|c|c|c|c|}
\hline & $V_{95 \%}$ to $V_{107 \%}$ (\%) (higher is better) & $\mathrm{D}_{5 \%} / \mathrm{D}_{95 \%}$ (lower is better) & HTCI (higher is better) & $\mathrm{CN}$ (higher is better) \\
\hline \multicolumn{5}{|c|}{ Inner quadrants $(n=33)$} \\
\hline Electron & $88.7 \pm 1.6$ & $1.17 \pm 0.02$ & $0.82 \pm 0.03$ & $0.43 \pm 0.01$ \\
\hline Photon & $90.0 \pm 0.6$ & $1.12 \pm 0.01$ & $0.79 \pm 0.02$ & $0.42 \pm 0.01$ \\
\hline$p$ & 0.462 & 0.024 & 0.159 & 0.694 \\
\hline \multicolumn{5}{|c|}{ Outer quadrants $(n=39)$} \\
\hline Electron & $54.4 \pm 4.6$ & $2.96 \pm 0.75$ & $0.49 \pm 0.03$ & $0.28 \pm 0.02$ \\
\hline Photon & $93.7 \pm 1.8$ & $1.07 \pm 0.05$ & $0.68 \pm 0.02$ & $0.37 \pm 0.03$ \\
\hline$p$ & $<0.001$ & 0.015 & $<0.001$ & $<0.001$ \\
\hline
\end{tabular}


Table 3

Dose to the OARs according to the location of the tumor

\begin{tabular}{|c|c|c|c|c|c|c|c|}
\hline & $\mathrm{V}_{100 \% \text { breast }}(\%)$ & $\mathrm{V}_{50 \% \text { breast }}(\%)$ & $\mathrm{V}_{25 \% \text { breast }}(\%)$ & $\mathrm{V}_{40 \% \text { lung }}(\%)$ & $\mathrm{V}_{10 \% \text { opp breast }}(\%)$ & $\mathrm{V}_{50 \% \text { heart }}(\%), n=22$ & $\mathrm{~V}_{20 \% \mathrm{LAD}}(\%), n=22$ \\
\hline \multicolumn{8}{|c|}{ Inner quadrants $(n=33)$} \\
\hline Electron & $1.6 \pm 0.3$ & $12.4 \pm 1.2$ & $17.0 \pm 1.5$ & $4.1 \pm 0.6$ & $0.0 \pm 0.0$ & $0.3 \pm 0.1$ & $24.5 \pm 5.7$ \\
\hline Photon & $1.9 \pm 0.5$ & $17.3 \pm 1.9$ & $34.4 \pm 2.0$ & $4.0 \pm 0.6$ & $11.0 \pm 2.5$ & $1.9 \pm 0.7$ & $29.6 \pm 6.6$ \\
\hline$p$ & 0.462 & 0.001 & $<0.001$ & 0.854 & $<0.001$ & 0.018 & 0.273 \\
\hline \multicolumn{8}{|c|}{ Outer quadrants $(n=39)$} \\
\hline Electron & $7.8 \pm 0.9$ & $18.9 \pm 1.3$ & $25.3 \pm 1.5$ & $2.7 \pm 0.5$ & $0.0 \pm 0.0$ & $0.3 \pm 0.1$ & $5.4 \pm 2.2$ \\
\hline Photon & $3.3 \pm 0.4$ & $25.7 \pm 1.3$ & $43.1 \pm 1.9$ & $4.5 \pm 0.6$ & $0.9 \pm 0.5$ & $0.2 \pm 0.1$ & $1.6 \pm 0.9$ \\
\hline$p$ & $<0.001$ & $<0.001$ & $<0.001$ & 0.007 & $<0.105$ & 0.639 & 0.097 \\
\hline
\end{tabular}

was not significantly different among the treatment groups. No breast edema or skin atrophy was detected. It can be stated that the relatively low rate of side effects in our study is owing to the use of small-dose fractions and irradiated volumes. The length of the follow-up time of this pilot study has been acceptable for the consideration of both the efficacy and the toxicity end points. For the evaluation of the final cosmetic outcome, nonetheless, a longer observation of up to 10 to 15 years would be needed.

For APBI, the most common teletherapy regimen is $10 \times$ $3.85 \mathrm{~Gy}, 2$ fractions per day. ${ }^{4}$ We used alternative fractionation, with special regard to the high skin dose in case of electron irradiation. Indeed, acute and late skin reactions were more frequent in the electron arms, and we believe that the daily dose of $3 \mathrm{~Gy}$ should not be exceeded if APBI is given with electron beams. The advantages of electron and photon irradiation may be simultaneously used in the mixed modality approach. In certain PBI cases, the dose distribution and OAR exposure may be optimized by the combination of photon and electron fields. ${ }^{23-25}$

Only 1 contemporary study has reported the use of sole electron fields for PBI. ${ }^{26,27}$ Altogether 40 patients with relatively small breasts received electron irradiation of a dose of $25 \times 2 \mathrm{~Gy}$; this technique resulted in the lowest rate (17.5\%) of fat necrosis of grade I to III, as compared with that in the brachytherapy and WBI arms. $^{26}$ None of our patients experienced symptomatic fat necrosis, which may be a consequence of the surgical and radiotherapy methods applied. In our experience, electron beams seem

\section{Table 4}

Skin and breast changes according to treatment arm. Acute dermatitis was graded at the end of the radiotherapy, whereas other side effects were evaluated $\geq 1$ year thereafter

\begin{tabular}{|c|c|c|c|c|c|}
\hline & \multicolumn{2}{|l|}{ Photon } & \multicolumn{2}{|l|}{ Electron } & \multirow{2}{*}{$\begin{array}{l}p \text {, electron } v s \\
\text { photon }\end{array}$} \\
\hline & $\begin{array}{l}25 \times 2 \mathrm{~Gy} \\
n=18\end{array}$ & $\begin{array}{l}13 \times 3 \mathrm{~Gy} \\
n=15\end{array}$ & $\begin{array}{l}25 \times 2 \mathrm{~Gy} \\
n=19\end{array}$ & $\begin{array}{l}13 \times 3 \mathrm{~Gy}, \\
n=20\end{array}$ & \\
\hline \multicolumn{6}{|c|}{ Acute dermatitis } \\
\hline $\begin{array}{l}\text { Grade } \\
\text { I }\end{array}$ & $2(11.1 \%)$ & $3(20 \%)$ & $11(57.9 \%)$ & $7(35 \%)$ & 0.001 \\
\hline $\begin{array}{l}\text { Grade } \\
\text { II }\end{array}$ & $0(0 \%)$ & $1(6.7 \%)$ & $2(10.5 \%)$ & $4(20 \%)$ & \\
\hline \multicolumn{6}{|c|}{ Hyperpigmentation } \\
\hline $\begin{array}{l}\text { Grade } \\
\text { I }\end{array}$ & $0(0 \%)$ & $0(0 \%)$ & $3(15.8 \%)$ & $1(5 \%)$ & 0.095 \\
\hline \multicolumn{6}{|c|}{ Teleangiectasia } \\
\hline $\begin{array}{l}\text { Grade } \\
\text { I }\end{array}$ & $0(0 \%)$ & $0(0 \%)$ & $3(15.8 \%)$ & $4(20 \%)$ & 0.048 \\
\hline \multicolumn{6}{|c|}{ Subcutaneous/breast fibrosis } \\
\hline $\begin{array}{l}\text { Grade } \\
\text { I }\end{array}$ & $0(0 \%)$ & $1(6.7 \%)$ & $0(0 \%)$ & $0(0 \%)$ & 0.408 \\
\hline \multicolumn{6}{|c|}{ Breast pain } \\
\hline $\begin{array}{l}\text { Grade } \\
\text { I }\end{array}$ & $2(11.1 \%)$ & $6(40 \%)$ & $4(21 \%)$ & $3(15 \%)$ & 0.273 \\
\hline
\end{tabular}

advantageous in approximately half of the cases with superficial and small PTVs, typical for inner-quadrant tumors.

\section{Conclusions}

In everyday practice, for PBI, we recommend CRT with photons or electrons according to the individual anatomic and geographic situation of the tumor bed. The fractionation schedule of $13 \times 3 \mathrm{~Gy}$ seems as feasible as $25 \times 2$ Gy.

\section{References}

1. Early Breast Cancer Trialists' Collaborative Group (EBCTCG), Darby, S.; McGale, P.; et al. Effect of radiotherapy after breast-conserving surgery on 10-year recurrence and 15-year breast cancer death: meta-analysis of individual patient data for 10,801 women in 17 randomised trials. Lancet. 378:1707-16; 2011.

2. Klepczyk, L.C.; Keene, K.S.; De Los Santos, J.F. Accelerated partial breast irradiation for early-stage breast cancer: Controversies and current indications for use. Curr. Treat. Options Oncol. 14:51-65; 2013.

3. Barry, M.; Ho, A.; Morrow, M. The evolving role of partial breast irradiation in early-stage breast cancer. Ann. Surg. Oncol. 20:2534-40; 2013.

4. Offersen, B.V.; Overgaard, M.; Kroman, N.; et al. Accelerated partial breast irradiation as a part of breast conserving therapy of early breast cancer: A systematic review. Radiother. Oncol. 90:1-13; 2009.

5. Smith, B.D.; Arthur, D.W.; Buchholz, T.A.; et al. Accelerated partial breast irradiation consensus statement from the American Society for Radiation Oncology (ASTRO). Int. J. Radiat. Oncol. Biol. Phys. 74:987-1001; 2009.

6. Polgár, C.; Van Limbergen, E.; Pötter, R.; et al. Patient selection for accelerated partial-breast irradiation (APBI) after breast-conserving surgery: recommendations of the Groupe Européen de Curiethérapie-European Society for Therapeutic Radiology and Oncology (GEC-ESTRO) breast cancer working group based on clinical evidence (2009). Radiother. Oncol. 94:264-73; 2010.

7. Shah, C.; Vicini, F.; Wazer, D.E.; et al. The American Brachytherapy Society consensus statement for accelerated partial breast irradiation. Brachytherapy. 12:267-77; 2013.

8. Lei, R.Y.; Leonard, C.E.; Howell, K.T.; et al. Four-year clinical update from a prospective trial of accelerated partial breast intensity-modulated radiotherapy (APBIMRT). Breast Cancer Res. Treat. 140:119-33; 2013.

9. Lewin, A.A.; Derhagopian, R.; Saigal, K.; et al. Accelerated partial breast irradiation is safe and effective using intensity-modulated radiation therapy in selected early-stage breast cancer. Int. J. Radiat. Oncol. Biol. Phys. 82:2104-10; 2012.

10. Patel, R.R.; Becker, S.J.; Das, R.K.; et al. A dosimetric comparison of accelerated partial breast irradiation techniques: Multicatheter interstitial brachytherapy, three-dimensional conformal radiotherapy, and supine versus prone helical tomotherapy. Int. J. Radiat. Oncol. Biol. Phys. 68:935-42; 2007.

11. Qiu, J.J.; Chang, Z.; Horton, J.K.; et al. Dosimetric comparison of 3D conformal, IMRT, and V-MAT techniques for accelerated partial-breast irradiation (APBI). Med. Dosim. 39:152-8; 2014.

12. Jagsi, R.; Ben-David, M.A.; Moran, J.M.; et al. Unacceptable cosmesis in a protocol investigating intensity-modulated radiotherapy with active breathing control for accelerated partial-breast irradiation. Int. J. Radiat. Oncol. Biol. Phys. 76:71-8; 2010

13. Liss, A.L.; Ben-David, M.A.; Jagsi, R.; et al. Decline of cosmetic outcomes following accelerated partial breast irradiation using intensity modulated radiation therapy: Results of a single-institution prospective clinical trial. Int. J. Radiat. Oncol. Biol. Phys. 89:96-102; 2014.

14. Hepel, J.T.; Tokita, M.; MacAusland, S.G.; et al. Toxicity of three dimensional conformal radiotherapy for accelerated partial breast irradiation. Int. J. Radiat. Oncol. Biol. Phys. 75:1290-6; 2009.

15. Olivotto, I.A.; Whelan, T.J.; Parpia, S.; et al. Interim cosmetic and toxicity results from RAPID: A randomized trial of accelerated partial breast irradiation using three-dimensional conformal external beam radiation therapy. J. Clin. Oncol. 31:4038-45; 2013. 
16. Wolmark N. Curran W.J.; Vicini F.; et al. Response to "Unacceptable cosmesis in a protocol investigating intensity-modulated radiotherapy with active breathing control for accelerated partial-breast irradiation" (Int J Radiat Oncol Biol Phys 2010;76:71-78) and "Toxicity of three-dimensional conformal radiotherapy for accelerated partial breast irradiation" (Int J Radiat Oncol Biol Phys 2009;75:1290-1296). Int. J. Radiat. Oncol. Biol. Phys. 77:317; 2010.

17. Shaikh, A.Y.; LaCombe, M.A.; Du, H.; et al. Accelerated partial breast irradiation using once-daily fractionation: analysis of 312 cases with four years median follow-up. Radiat. Oncol. 7:17; 2012.

18. Varga, Z.; Hideghéty, K.; Mezo, T.; et al. Individual positioning: a comparative study of adjuvant breast radiotherapy in the prone versus supine position. Int. J. Radiat. Oncol. Biol. Phys. 75:94-100; 2009.

19. Yang, T.J.; Tao, R.; Elkhuizen, P.H.; et al. Tumor bed delineation for external beam accelerated partial breast irradiation: A systematic review. Radiother. Oncol. 108:181-9; 2013.

20. Varga, Z.; Cserháti, A.; Rárosi, F.; et al. Individualized positioning for maximum heart protection during breast irradiation. Acta Oncol. 53:58-64; 2014.

21. Feuvret, L.; Noël, G.; Mazeron, J.J.; et al. Conformity index: A review. Int. J. Radiat. Oncol. Biol. Phys. 64:333-42; 2006.
22. Shiu, A.R.; Hogstrom, K.R. Dose in bone and tissue near bone-tissue interface from electron beam. Int. J. Radiat. Oncol. Biol. Phys. 21:695-702; 1991.

23. Bourgier, C.; Acevedo-Henao, C.; Dunant, A.; et al. Higher toxicity with 42 Gy in 10 fractions as a total dose for 3D-conformal accelerated partial breas irradiation: Results from a dose escalation phase II trial. Radiat. Oncol. 7:141; 2012.

24. El Nemr, M.; Heymann, S.; Verstraet, R.; et al. Mixed modality treatment planning of accelerated partial breast irradiation: To improve complex dosimetry cases. Radiat. Oncol. 6:154; 2011.

25. Palma, B.A.; Sánchez, A.U.; Salguero, F.J.; et al. Combined modulated electron and photon beams planned by a Monte-Carlo-based optimization procedure for accelerated partial breast irradiation. Phys. Med. Biol. :1191-202; 2012.

26. Lövey, K.; Fodor, J.; Major, T.; et al. Fat necrosis after partial-breast irradiation with brachytherapy or electron irradiation versus standard whole-breast radiotherapy-4-Year results of a randomized trial. Int. J. Radiat. Oncol. Biol. Phys. 69:724-31; 2007.

27. Polgár, C.; Fodor, J.; Major, T.; et al. Breast-conserving therapy with partial or whole breast irradiation: ten-year results of the Budapest randomized trial Radiother. Oncol. 108:197-202; 2013. 